\title{
Eficacia de un programa de intervención para la mejora del clima escolar: algunos resultados
}

\author{
Sánchez, A. $\mathbf{M}^{1}$, \\ Rivas, M. $\mathbf{T}^{2}$. \\ Trianes, M. V. ${ }^{1}$ \\ ${ }^{1}$ Departamento de Psicología Evolutiva y de la Educación, \\ ${ }^{2}$ Departamento de Psicología Básica, Psicobiología y Metodología de las \\ Ciencias del Comportamiento, \\ Universidad de Málaga
}

\section{España}




\section{Resumen}

Este estudio versa sobre los resultados de la aplicación del Programa de Desarrollo Social y Afectivo (Trianes y Muñoz, 1994; Trianes, 1996), a lo largo de cuatro años, en un colegio de Atención Educativa Preferente de la capital de Málaga. La intervención se dirige a mejorar el clima de aula y centro como camino para la prevención de violencia escolar. Se describe el enfoque de intervención utilizado, los materiales del Programa y resultados obtenidos en relación a variables de contexto (clima de la clase). La discusión plantea la oportunidad de mejorar las relaciones interpersonales y clima social del centro, como eje de la intervención preventiva de violencia escolar.

Palabras Clave: clima escolar, programa intervención y, prevención, competencia social, variables del contexto 


\section{Introducción}

La competencia social se ha establecido como un área clave de salud mental y bienestar psicológico, en jóvenes y adultos. Ha propiciado el desarrollo de programas dirigidos a prevenir problemas de inadaptación social y a promover competencia social y relaciones interpersonales satisfactorias. La promoción de competencia social guarda relación con la actual concepción de la educación escolar como práctica social y socializadora. Esta tiene como objetivo promover el desarrollo social y personal del alumno/a (Coll, 1990).

En esta línea de trabajo se han publicado programas de promoción de competencia social dado que esta vía ha sido vista como uno de los caminos más efectivos para prevenir conflictos y violencia en los centros escolares. La enseñanza de estrategias y procedimientos para mejorar las relaciones sociales asegura que todo el alumnado adquiera las habilidades sociales, incluso aquellos, que por presentar riesgos de inadaptación, podrían no desarrollar o hacerlo muy lentamente, dichas habilidades.

En los últimos veinte años, son muchos los programas que han surgido en esta línea de intervención, entre ellos: "Habilidades Sociales en la Infancia" (Michelson, Sugai, Wood, y Kazdin, 1983); "Aprendizaje Estructurado" (Goldstein, Sprafkin, Gershaw y Klein, 1980); "Think Aloud" (Camps y Bash, 1981); "La Solución de Problemas Interpersonales" (Spivack y Shure, 1974); "Enseñanza de Habilidades de Interacción Social" (P.E.H.I.S., de Monjas Casares, 1993); "Programa para Favorecer el Desarrollo de la Tolerancia en Contextos Etnicamente Heterogéneos"(Díaz Aguado,1992); "Aprender a Vivir Juntos" (Borrego y Morales, 1991); "El Programa de Educación Social y Afectiva"(Trianes y Muñoz, 1994; Trianes, 1996); "Educar en la Competencia Social. Un Programa para la Tutoría con Adolescentes" (Moraleda, 1998); "Aprender a Ser Personas y a Convivir" (Trianes y Fernández-Figarés, 2001), "Ser Persona y Relacionarse. Habilidades Cognitivas y Sociales y Crecimiento Moral" (Segura, 2002).

Estos programas se pueden dividir en tres tipos básicos: los centrados en la persona; los centrados en la situación o contexto (Cowen, 1985) y los centrados en la interacción persona/contexto (Felner y Felner, 1989). Los programas centrados en la persona pretenden incidir y provocar cambios directamente en los individuos, desarrollando competencias individuales; mientras que los programas centrados en el entorno o ambiente inciden de 
manera indirecta sobre los individuos, modificando su entorno, es decir la intervención se dirige hacia el entorno. El enfoque más actual, se centra en la interacción individuocontexto.

Los programas centrados en la interacción individuo-contexto han adoptado metas que se refieren a la mejora de competencias a través de cambios en la relación docente o en el tratamiento del centro de los problemas interpersonales. Partiendo de enfoques pioneros como el de Spivack y Shure (1974) se ha pretendido la formación de los escolares en solución de problemas interpersonales como vía más popular para la prevención de problemas futuros así como para la mejora de adaptación social y escolar. Otros trabajos también en los años 80 difundieron materiales para promover competencias en alumnado con riesgos iniciales contribuyendo así a una mejor adaptación y salud mental de los mismos.

Actualmente prevalece el enfoque centrado en la interacción. Surge del modelo ambiental y ecológico. Asume que el riesgo radica en la interacción entre el niño/a y las características del contexto en el que se desarrolla. De manera que los déficits de habilidades no van a estar en función de las características inherentes del niño/a, sino de su vulnerabilidad ante el contexto particular (físico y social) que le rodea. Así, este tipo de programas pretende eliminar las causas o mediadores del desajuste modificando el ambiente. Felner y Felner (1989) consideran que son programas que tienen como objetivos, proporcionar recursos en el entorno para facilitar el desarrollo de habilidades y competencias y producir cambios y modificaciones de condiciones que son potencialmente peligrosas. Al mismo tiempo pretenden la promoción de competencias en los individuos para que aprovechen las mejoras en el entorno. Este es el modelo transaccional, que concibe el riesgo como combinación de condiciones personales y ambientales. De manera que los programas pueden centrarse en uno o ambos aspectos de la transacción.

En el sistema escolar, este enfoque supone analizar como pueden ser reorganizados los elementos del entorno-escuela, para que permitan el desarrollo de competencias. Al respecto, Lemle (1976) establece tres caminos diferentes o formas de considerar variables ambientales del contexto escolar.

- El enfoque que pretende mejoras en el currículum escolar. Ha pretendido introducir cambios en forma de programas, en el curriculum ordinario, sobre todo en infantil y 
primaria, con la participación potencial de las familias. Así, se han publicado programas dirigidos a la enseñanza de habilidades comportamentales conectadas a situaciones especificas (Michelson et al.,1983, Goldstein et al., 1980). Otros programas enseñan estrategias generales de pensamiento no vinculadas a situaciones concretas y que asegurarían la generalización de los comportamientos aprendidos (Díaz Aguado, 1990, Gesten y Weissberg, 1979, Monjas, 1993, Pelechano, 1991, Spivak y Shure, 1974; Trianes y Muñoz, 1994, y Trianes, 1996). Estos programas despiertan mayor interés, hoy, por parte de los educadores.

- El enfoque que propone modificaciones ambientales, utiliza los propios recursos de la escuela de manera sistemática y organizada y promueve mejoras como aulas abiertas, organización cooperativa de la clase, tutorías de iguales, ligas de alumnos, etc.

- Y el enfoque centrado en la formación de profesores, supone implicar al profesorado en la implantación y seguimiento de programas. Asume como premisa que el profesor/a debe ser entrenado en las habilidades que va enseñar. Ello aumenta su capacidad profesional ya que le proporciona nuevos recursos para su relación docente diaria. Son muchos los programas que atribuyen al profesor/a un papel central, ya sea como mediador/a, como modelo y/o agente de control. En esta línea, se ha detectado un número de variables relevantes en la personalidad y comportamiento del profesorado cuya optimización puede redundar en una mayor efectividad de los programas.

En el presente trabajo se ha utilizado un enfoque de prevención basado en la interacción individuo/contexto y concretamente, en la formación del profesorado. Nuestro programa se dirige a enseñar habilidades y competencias sociales para mejorar el clima social de la clase. Con ello se previene la resolución destructiva de conflictos interpersonales que pueda originar escaladas de violencia. Dentro de los resultados de efectividad del programa, se presentan aquí los relativos a la evaluación del clima de clase, cuya mejora ha sido un objetivo importante en el programa. 


\section{Método}

Participantes

La muestra la componen 44 alumnos/as (27 alumnos y 17 alumnas) que han sido evaluados durante cuatro años de su escolarización obligatoria. Son alumnos/as que pertenecen a dos colegios públicos de Zona de Atención Educativa Preferente de la capital de Málaga (según la clasificación establecida por la Consejería de Educación de la Junta de Andalucía).

La media de edad, rango y desviación típica en años de estos/as alumnos/as por años de investigación se muestra en la Tabla 1.

Tabla .1. Edad de los alumnos/as por años de investigación.

\begin{tabular}{|c|c|c|c|c|}
\cline { 2 - 5 } \multicolumn{1}{c|}{} & \multicolumn{4}{|c|}{} \\
\hline EDAD & $\begin{array}{c}1^{\text {ER }} \text { AÑO DE } \\
\text { INVESTIGACIÓN }\end{array}$ & $\begin{array}{c}2^{\circ} \text { AÑO DE } \\
\text { INVESTIGACIÓN }\end{array}$ & $\begin{array}{c}3^{\circ} \text { AÑO DE } \\
\text { INVESTIGACIÓN }\end{array}$ & $\begin{array}{c}4^{\circ} \text { AÑO DE } \\
\text { INVESTIGACIÓN }\end{array}$ \\
\hline MEDIA & $\mathbf{1 0 , 3}$ & 11,3 & 12,3 & 13,3 \\
\hline DT & $\mathbf{0 , 8 5}$ & 0,95 & 0,95 & 0,91 \\
\hline RANGO & $9-12$ & $10-13$ & $11-14$ & $12-15$ \\
\hline
\end{tabular}

Los centros escolares que accedieron a participar de manera voluntaria durante todo el tiempo que duró la investigación se asignaron a dos grupos siguiendo los criterios que se especifican a continuación:

a) Grupo Intervenido (GI), constituido por alumnos/as de un colegio de zona de Atención Preferente de la capital de Málaga a los que se le aplica el programa (Trianes y Muñoz, 1994; Trianes, 1996), durante tres años consecutivos y un año de seguimiento. La variable sexo en este grupo se distribuyó, en quince niños y siete niñas.

b) Grupo No Intervenido (GNI), formado igual que el grupo Intervenido por alumnos/as de un colegio de zona de atención Preferente de la Málaga capital que no reciben ningún tipo de intervención. La distribución de los sujetos según el sexo ha sido doce niños y diez niñas 


\section{Instrumentos y materiales}

El Instrumento de evaluación utilizado ha sido la Escala de Clima Social: Centro escolar (Tricket y Moss, 1984) contestada por el alumnado. Esta prueba se ha adaptado a un formato de más fácil aplicación, teniéndose en cuenta, solamente, tres subescalas:

1. Control. Grado en que el profesor/a es estricto en sus controles sobre el cumplimiento de las normas y en la penalización de los infractores. Tiene 20 ítems

2. Claridad. Importancia que se le da al establecimiento y seguimiento de normas claras y apercibimiento por parte de los/as alumnos/as de las consecuencias de su incumplimiento. Tiene 20 ítems.

3. Innovación. Grado en que los/as alumnos/as contribuyen a planear las actividades escolares y la variedad y cambios que introduce el profesor/a con nuevas técnicas y estímulos a la creatividad del alumno.

El programa de intervención utilizado ha sido el Programa de Desarrollo Social y Afectivo (Trianes y Muñoz, 1994; Trianes, 1996). Se basa en el principio de que promover la competencia social y la resolución constructiva de conflictos interpersonales redunda en la prevención de violencia escolar. Consta de tres módulos y cincuenta y cuatro actividades. Los objetivos que se establecen por módulo son: a) Modulo I, "Mejorar el clima de clase"; b) Modulo II, "Solucionar problemas con los demás sin pelearnos" y c) Modulo III, "Aprender a ayudar y a cooperar".

Los procedimientos centrales utilizados en las distintas actividades presentan una larga tradición en la Psicología de la Educación y del Desarrollo, concretamente, han sido: la reflexión y discusión, la participación activa del alumno/a y el entrenamiento en habilidades de solución de conflictos interpersonales.

\section{Procedimiento}

Se ha adoptado como criterio de selección de los grupos, la participación voluntaria de profesores/as tutores/as en la Formación y Aplicación del Programa (GI) y la no participación de profesores/as tutores/as en la formación y aplicación de dicho programa (GNI). Inicialmente, la participación voluntaria del profesorado surgió de forma natural, concretamente, a raíz de la demanda inicial, planteada por un profesor y una profesora tutores de un colegio público de Zona de Atención Preferente, relativa a la solución de 
problemas de disciplina que estaba afectando a la vida en clase. Esta demanda se hizo a la orientadora del Equipo de Orientación de la Zona Palma-Palmilla (EPOE), quien se puso en contacto con nuestro equipo de Investigación para recibir asesoramiento con el objetivo de resolver dichos problemas de disciplina. Tras el primer contacto con la orientadora, el grupo de investigación realiza un análisis inicial de dicha demanda y propone la aplicación del Programa de Educación Social y Afectiva (PESA) (Trianes y Muñoz, 1994; Trianes, 1996) a dicho profesorado. Transcurrido el primer año de aplicación del programa y tras divulgarse la experiencia de dos profesores con el programa, al año siguiente solicitan continuar la aplicación de éste, aunque el segundo año la petición la realiza todo el profesorado que impartía docencia al alumnado que había empezado el entrenamiento con el programa el año anterior. La intervención fue apoyada por el personal SOEV de la zona, quien participó activamente en la formación y apoyo al profesorado durante el desarrollo de las actividades en las clases.

El diseño que hemos desarrollado a lo largo de los cuatro años ha mantenido como estructura básica, tres fases de intervención con el Programa abarcando formación del profesorado e implantación del programa en el aula y cinco fases de evaluación en las que se cumplimentan pruebas por parte de los alumnos y por parte de los profesores. Como se aprecia en la Tabla 2, los momentos de evaluación y los módulos del programa aplicados por año de investigación han sido:

El primer año y coincidiendo con el inicio del curso escolar se realiza una evaluación "previa" a la implantación del programa que hemos denominado Medida Pre. Luego, a lo largo del curso, se desarrolló la primera fase de intervención, aplicándose el Modulo I de dicho programa, y al finalizar dicho curso escolar tuvo lugar una evaluación "a posteriori" a la aplicación del programa, denominada Medida Post 1.

En el segundo año de la investigación se realizo la segunda fase de intervención, centrada de nuevo en el Modulo I del Programa. La razón de repetir el Modulo I estaba justificada por el hecho de que se incorporaron nuevos profesores al desarrollo del programa y necesitaban la formación en dicho modulo. Al finalizar el curso escolar, se realizo su correspondiente evaluación "a posteriori" a la aplicación del Modulo I, identificada como Medida Post 2. 
Tabla 2. Temporización de la Intervención y Evaluación.

\begin{tabular}{|c|c|c|c|c|}
\cline { 2 - 5 } \multicolumn{1}{c|}{} & \multicolumn{3}{c|}{ AÑOS DE LA INVESTIGACIÓN } \\
\cline { 2 - 5 } \multicolumn{1}{c|}{ EVALUACIÓN } & $1^{\circ}$ año & $2^{\circ}$ año & $3^{\circ}$ año & $4^{\circ}$ año \\
\hline $\begin{array}{c}\text { MEDIDA PRE. } \\
\text { MEDIDA POST1 } \\
\begin{array}{c}\text { DELL } \\
\text { PROGRAMA }\end{array}\end{array}$ & $\begin{array}{c}\text { MEDIDA } \\
\text { POST2 }\end{array}$ & $\begin{array}{c}\text { MEDIDA } \\
\text { POST3 }\end{array}$ & $\begin{array}{c}\text { MEDIDA } \\
\text { SEGUIMIENTO }\end{array}$ \\
\hline
\end{tabular}

En el tercer año de dicha investigación se desarrolló la tercera fase de intervención con la aplicación del Modulo II del programa y al final de curso se realizó su correspondiente evaluación "a posteriori” a la aplicación del Modulo II, identificada como Medida Post 3.

Finalmente, y en el cuarto año se realizó el seguimiento de la intervención, con su correspondiente fase de evaluación al finalizar el curso escolar que se denominó Seguimiento.

\section{Análisis de Resultados}

En primer lugar, se debe hacer constar que, a lo largo de la exposición de los resultados, el grupo GNI, se interpreta con relación a los distintos momentos de la aplicación del programa aunque esto sólo se toma como referencia porque este grupo no ha recibido aplicación del programa.

Con objeto de analizar el efecto de la aplicación del programa a nivel inferencial se han aplicado análisis ANCOVA Y ANOVA con el paquete estadístico SPSS (Versión 12.0) y las pruebas a posteriori (Análisis de tendencias y test a posteriori LSD de Fisher) con el programa STATISTICA. El Análisis de Estadística Inferencial ha permitido analizar las diferencias estadísticamente significativas (Entre Grupos, Intra-Grupos e Interacción) en los distintos factores de la prueba tras la aplicación del programa. Con objeto de analizar el efecto de la aplicación del programa en los distintos momentos se ha utilizado:

Análisis de la Covarianza (ANCOVA). Este va a permitir ajustar las diferencias que existen antes de la intervención entre los grupos (GNI y GI) en este estudio no aleatorizado. 
En caso de que no se cumpla el supuesto de la Homogeneidad de la Regresión y se verifiquen los supuestos de Homoscedasticidad ó Esfericidad se utiliza Análisis de la Varianza de Medidas Repetidas (ANOVA) de las puntuaciones diferencia en cada momento de la evaluación con respecto a la medida Pre-Intervención.

Cuando los resultados de los análisis anteriores lo requieren, se aplica una las dos pruebas a posteriori que siguen:

2.1) Análisis de Tendencias, para determinar si hay una tendencia (lineal, cuadrática, etc.)

2.2) Test a posteriori LSD de Fisher, para determinar los momentos de la intervención en los que se dan las diferencias estadísticamente significativas.

\section{Resultados}

El análisis ANCOVA/ANOVA efectuado arroja tres tipos de información. En primer lugar se presentan las diferencias obtenidas en el factor Grupo, es decir, en ambos grupos (GNI y GI) a lo largo de la dimensión temporal. En segundo lugar se presentan las diferencias obtenidas en el factor Tiempo, o sea las diferencias encontradas a lo largo de la aplicación del programa independientemente del grupo. En tercer lugar se exponen las diferencias debidas a la Interacción de ambos factores.

1) Diferencias significativas entre el GNI y GI en los tres factores de dicha prueba: Control $\left(F_{(1,41)}=5,96, \mathrm{P}<0,05\right)$, Claridad $\left(F_{(1,41)}=7,26, \mathrm{P}<0,05\right)$ e Innovación $\left(F_{(1,41)}=9,55 \mathrm{P}<0,05\right)$, concretamente:

El factor Control presenta diferencias significativas en el momento Post2 y en el Seguimiento, presentando GI menor nivel de Control que el GNI. (Ver Figura 1.) 
Figura 1. Medias del factor Control en los distintos momentos

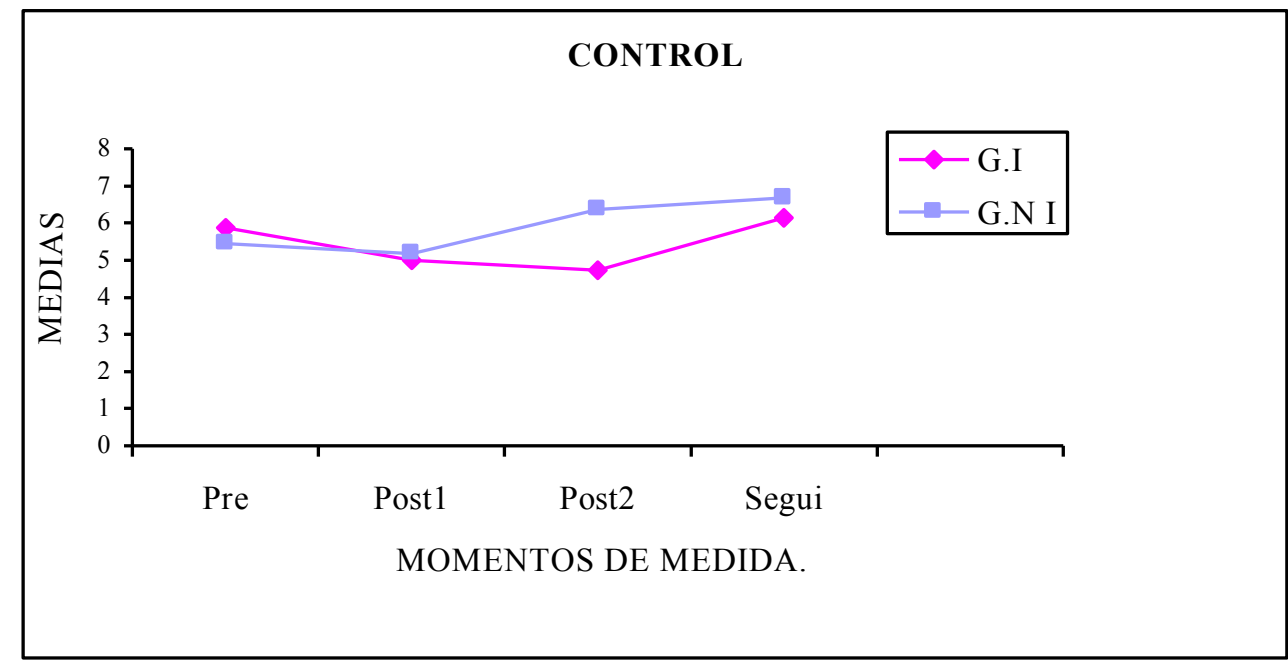

El factor Claridad presenta diferencias en el momento Post1 y en el momento Post2, presentando el GI un menor nivel de Claridad que el G NI. (Ver Figura 2.)

Figura 2. Medias del factor Claridad en los distintos momentos

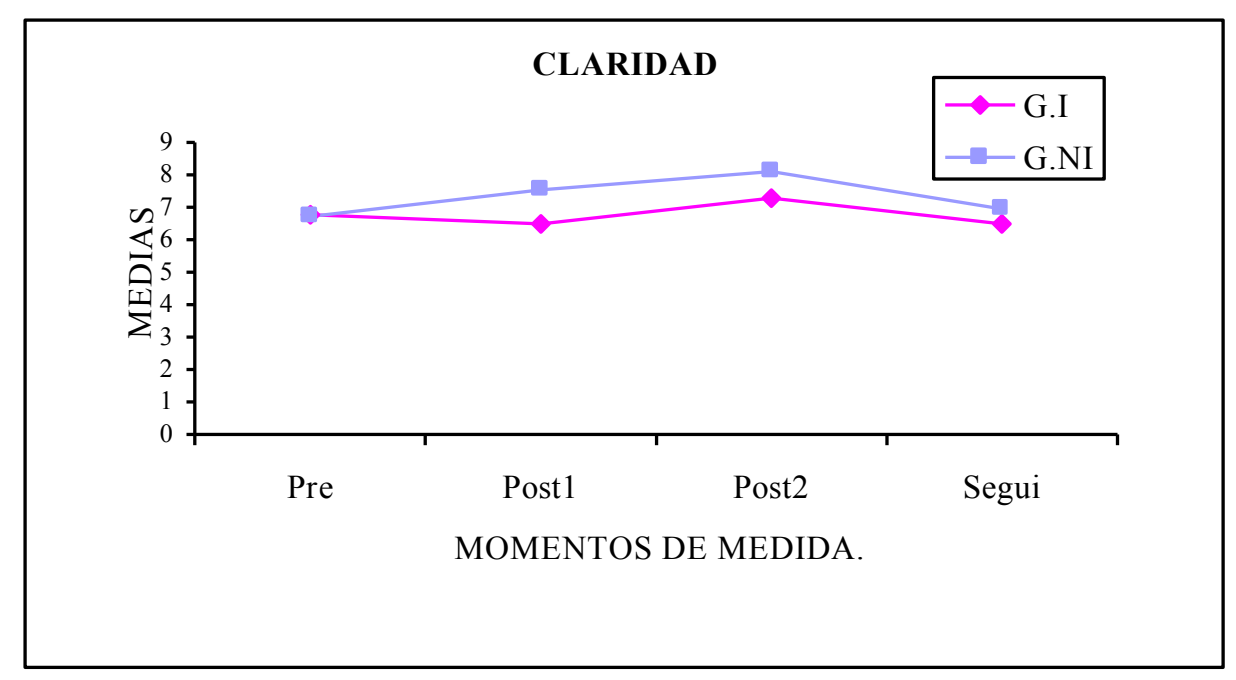

Y el factor Innovación presenta diferencias en los momentos Post1 y Seguimiento, presentando el GI menor nivel de Innovación que el GNI. (Ver Figura 3) 
Figura 3. Medias del factor Innovación en los distintos momentos

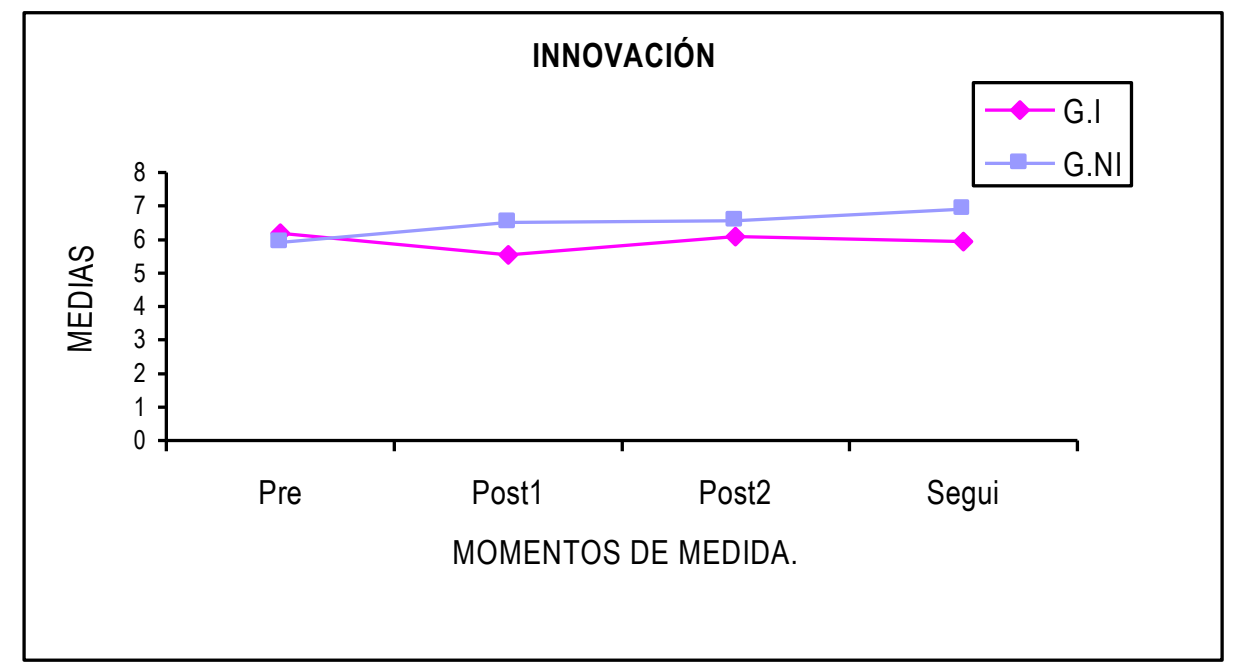

2) Diferencias a lo largo de la Aplicación del Programa en los factores Control $\left(F_{(2,84)}=9,07, \mathrm{P}<0,05\right)$ y Claridad $\left(F_{(2,84)}=5,52, \mathrm{P}<0,05\right)$. Concretamente, el factor Control presenta diferencias significativas en el:

- GI en los momentos Post2 y Seguimiento y en los momentos Post1 y Seguimiento, presentando este grupo mayor nivel de Control en el Seguimiento que tras aplicar el programa el primer y segundo año (Ver Figura 1).

- GNI en los momentos Post1 y Post2, presentando este grupo un mayor nivel de Control el segundo año que el primer año de aplicación del programa. El GNI también presenta mayor nivel de Control en el Seguimiento que en el Post1. (Ver figura 1)

En relación con el factor Claridad encontramos diferencias significativas en el:

- GI en los momentos Post1 y Post2, presentando mayor nivel de Claridad el segundo año de aplicación del programa que el primero. El GI también muestra menor nivel de Claridad en el Seguimiento que el segundo año de aplicación del programa. (Ver Figura 2)

3) No hay diferencias significativas debidas a la Interacción (GrupoxFactor) en ningún factor de la prueba: Control $\left(F_{(2,84)}=2,90, \mathrm{P}>0,05\right)$; Claridad $\left(F_{(2,84)}=0,49, \mathrm{P}>0,05\right)$ e Innovación $\left(F_{(2,84)}=0,38 \mathrm{P}>0,05\right)$. 


\section{Discusión}

Los resultados indican que en el factor Control disminuye la puntuación media en el GI con respecto al GNI después de aplicar el segundo año el programa y en el seguimiento. Dicho factor presenta contenidos referidos a conductas directivas por parte del profesorado concibiéndose la imposición de las normas desde la autoridad. El resultado obtenido puede ser atribuido a la intervención, en la que se promocionan comportamientos menos directivos por parte del profesorado, y mayor autogestión del alumnado. Precisamente, en el primer módulo del programa, aplicado el primer y segundo año, se trabaja autogestión en el establecimiento de normas por parte del alumnado como vía para mejorar el clima de la clase.

Con respecto a la variable Claridad, el GI presenta una menor puntuación media en el segundo y tercer momento, siendo estas diferencias significativas. Su trayectoria, en general, es de menor puntuación. Esta variable también enfatiza la directividad del profesorado en su clase así como la Claridad en el establecimiento y Seguimiento de normas impuestas. El programa promociona otro tipo de comportamientos docentes, tendiendo a dar autonomía al grupo clase. El profesorado puede adoptar una estrategia más inductiva que directiva. Por tanto, este resultado lo consideramos también efecto del programa.

El factor Innovación presenta menor puntuación media en el GI que el GNI, en el segundo momento y en el Seguimiento. La interpretación la hacemos en el mismo sentido. Este patrón de resultados, que supone bajas puntuaciones debidas a la intervención coincide con lo que puede esperarse basándonos en la naturaleza de la prueba de Moos.

Se pone de manifiesto en estos resultados que la prueba utilizada, aunque ha sido ampliamente empleada para evaluar el clima de la clase, evalúa en realidad clima académico de la clase. Esta prueba fue seleccionada en este trabajo por prestigiosa en un área en la que existían escasas pruebas psicométricas para evaluar variables del contexto por parte del alumnado, aunque la mayor parte de sus subpruebas están dirigidas a evaluar climas académicos, es decir, la relación entre profesorado-alumnado en cuanto al desarrollo de las materias escolares. El estudio del clima de la clase recientemente se concreta en dos campos de estudio diferentes: clima académico y clima social de la clase.

El clima social de la clase es un concepto relativamente novedoso que se suele definir como la calidad de las interacciones entre estudiantes-profesorado y entre 
estudiantes-estudiantes (Emmons, Comer y Haynes, 1996), o también como la percepción, por parte de sus integrantes, alumnos/as y profesores/as, de bienestar personal, sentimientos positivos de sentirse aceptado y ser valioso para los demás en la convivencia diaria (Trianes, 2000). El clima académico, por su parte, se refiere a la extensión en la cual el entorno de aprendizaje estimula el esfuerzo y enfatiza la cooperación (Roeser y Eccles, 1998). El presente trabajo se expone los resultados de una intervención entre cuyos objetivos se encuentra la mejora del clima social de la clase y centro. Hoy nuestro grupo posee una prueba que evalúa clima social de la clase (Trianes, Blanca, de la Morena, Infante y Raya, 2006) que al inicio del presente trabajo no estaba disponible.

Para concluir, señalar que se ha presentado un programa para la mejora de la convivencia que presenta diversos objetivos relacionados en el ámbito de la competencia social y las relaciones sociales. El programa presenta resultados positivos (Sánchez, 2005; Trianes, Cardelle-Elawar, Blanca, y Muñoz, 2003). Trianes, Blanca, Muñoz, García, CardelleElawar y Infante, (2002) y Trianes, Muñoz. y Sánchez, (2001) le avalan como instrumento o recurso para el profesorado, mostrando una vía para intervenir preventivamente, antes de que la violencia sea ya grave, y reducir tensiones, mejorar relaciones interpersonales, redundando en el desarrollo profesional del docente, y en el bienestar, motivación e implicación del alumnado en la marcha del centro. 


\section{Referencias}

Borrego de Dios, C. y Morales, J. (1991). El desarrollo socio-personal como ámbito específico de contenidos escolares: Una propuesta educativa. Comunicación en el I seminario sobre desarrollo del curriculum escolar en el ámbito socio-personal. Sevilla: I.C.E.

Camps, B. W. y Bash, M. A. (1981). Think Aloud: Increasing social and cognitive skills. A problem-solving program for children. En D. P. Rathjen y J. P. Foreyt (Eds.), Social Competence: Intervention for Children and Adults. Champaing: Research Press.

Coll, C. (1990). Un marco de referencia psicológico para la educación escolar: la concepción constructivista del aprendizaje escolar y de la enseñanza. En C. Coll, J. Palacios y A. Marchesi (Comps.), Desarrollo psicológico y educación II. Psicología de la Educación. Madrid: Alianza Editorial.

Cowen, E. L. (1985). Person-centered approaches to primary prevention in mental health: Situation-focussed and competence enhancement. American Journal of Community Psychology, 13, 31-48.

Díaz-Aguado, M. J. (1990). Programa para el desarrollo de la "competencia social" en sujetos con inadaptación emocional. En J. M. Román Sánchez, Intervención Clínica y Educativa en el ámbito escolar, 172-187. Valencia: Promolibro. Serie Aplicada.

Díaz-Aguado, M. J. (1992). Programa para favorecer el desarrollo de la tolerancia en contextos étnicamente heterogéneos. Madrid:MEC.

Emmons, C. L., Comer, J. P., Haynes, N. M. (1996). Translating theory into practice: Comer's theory of school reform. En J. P. Comer, N. M. Haynes, E.Joyner y M. Ben-Avie (Eds.), Rallying the whole village. New York: Teachers College Press.

Felner, R. D. y Felner, T. Y. (1989). Primary Prevention Programs in the Educational Context: A Transactional-Ecological Framework to Analysis. En L. A. Bond y B. E. (Eds.), Primary Prevention and promotion in the Schools. California: Sage.

Gesten, E. L. y Weissberg, R. P. (1979). Social Problem solving training and prevention: Some good news and some bad news. Comunicación presentada en la Reunión Anual de la American Psychological Association. Nueva York.

Goldstein, A. P., Sprafkin, R. P., Gershaw, N. J. y Klein, P. (1980). Skill streaming the adolescent. Urbana, IL: Research Press (trad.español, 1989). 
Lemle, R. (1976). Primary prevention of psychological disorders in elementary and intermediate schools. Journal of Clinical Child Psychology, 5, 26-32.

Michelson, L., Sugai, D. P., Wood, R. P. y Kazdin, A. E. (1983). Social skills assesment and training with children. New York: Plenum Press (traducción castellano, 1985, Barcelona: Martínez Roca).

Monjas, M. I. (1993). Programa de enseñanza de habilidades de interacción social para niños y niñas de edad escolar. Valladolid: Trilce.

Moraleda, M. (1998). Educar en la Competencia Social. Un programa para la tutoría con Adolescentes. Madrid: CCS.

Pelechano, V. (1991). Inteligencia y habilidades interpersonales: un diseño de modelo de trabajo y algunas hipótesis contrastables. Psicologema, 5(10), 205-248.

Roeser, R. W. y Eccles, J. S. (1998). Adolescents' perceptions of middle school: Relation to longitudinal changes in academic and psychological adjustment. Journal of Research on Adolescence, 8, 123-158.

Sánchez, A, M. (2005). Una intervención psicoeducativa sobre competencia social en alumnado de 9 a 15 años: su evaluación con un diseño longitudinal. Tesis doctoral. Universidad deMálaga. (pendiente de publicación).

Segura, M. (2002). Ser persona y relacionarse. Habilidades cognitivas y sociales y crecimiento moral. Madrid: Narcea.

Spivack, G. y Shure, M. (1974). Social adjustment of young children. A cognitive approach to solving real life problems. San Francisco: Jossey Bass.

Trianes, M. V. (1996). Educación y Competencia Social. Un programa en el aula. Málaga: Aljibe.

Trianes, M. V. (2000). Violencia en contextos escolares. Málaga: Aljibe.

Trianes, M. V. y Muñoz, A. M. (1994). Programa de educación Social y Afectiva. Málaga: Puerta Nueva.

Trianes, M. V. y Fernández-Figarés, C. (2001). Aprender a ser persona y a convivir: un programa para secundaria. Bilbao: Descleé de Bower.

Trianes, M.V., Muñoz, A. y Sánchez, A. (2001). Educar la convivencia como prevención de violencia interpersonal: perspectiva de los profesores. Revista Interuniversitaria de Formación de Profesorado, 41, 73-93. 
Trianes, M.V., Blanca, M. J., Muñoz, A., García, B., Cardelle-Elawar, M. y Infante, L. (2002). Relaciones entre evaluadores de la competencia social en preadolescentes: profesores, iguales y autoinforme. Anales de Psicología, 18(2), 197-214.

Trianes, M.V., Cardelle-Elawar, M., Blanca, M.J. y Muñoz, A. (2003). Contexto social, género y competencia social autoevaluada en alumnos andaluces de 11-12 años. Revista Electrónica de Investigación Psicoeducativa y Psicopedagógica, 2(1), 3855.

Trianes, M.V., Blanca, M.J., de la Morena, L., Infante, L., y Raya, S. (2006). Un cuestionario para evaluar el clima social de la clase y centro. Psicothema, 18(2), 266-271.

Tricket, E. y Moos, R. H. (1984). Escala de Clima de Social. Madrid: TEA. 
[página en blanco por razones de paginación] 